\title{
A CYTOGENETIC SURVEY OF CONSECUTIVE LIVEBORN INFANTS-INCIDENCE AND TYPE OF CHROMOSOME ABNORMALITIES
}

\author{
Tohru MaEda, Michiko Ohno, Masumi Takada, \\ Yoshikatsu Kato,* Masato Nishida, * Toshiko JoBo,* \\ Hideo ADACHI,* and Akira TAGUCHI* \\ Department of Laboratory Medicine, Kitasato University School of Medicine, \\ Sagamihara, Kanagawa, Japan \\ *Department of Obstetrics and Gynecology, Kitasato University School of Medicine, \\ Sagamihara, Kanagawa, Japan
}

\begin{abstract}
Summary Cytogenetic investigations have been carried out with particular concern to the frequency of chromosome abnormalities in newborn infants, and this is a preliminary report of data derived from 2,626 consecutive liveborns (1,393 males and 1,233 females) which were collected in one hospital. Of these, 18 infants $(0.69 \%)$ were found to have a major chromosome abnormality. Eight infants $(0.30 \%)$ showed sex-chromosome abnormalities: two with $47, \mathrm{XYY}$, one with $47, \mathrm{XXX}$, one with $45, \mathrm{X} / 47, \mathrm{XXX}$ mosaicism, one with $46, \mathrm{XY} / 47, \mathrm{XYY}$ mosaicism, one with $45, \mathrm{X} / 46, \mathrm{XX}$ mosaicism, and a pair of twins with $45, \mathrm{X} / 46, \mathrm{XY} / 47, \mathrm{XYY}$ mosaicism. Ten infants $(0.38 \%)$ were carriers of autosomal abnormalities: three with 21-trisomy, one with 13-trisomy by translocation, three with a balanced $13 / 14$ translocation, two with a balanced 14/22 translocation and one with an extra small marker chromosome. Of chromosomally abnormal infants, 14 were phyiscally normal.
\end{abstract}

\section{INTRODUCTION}

Recently chromosome surveys of newborn infants have been undertaken in considerably large scale in various countries (Sergovich et al., 1969; Gerald and Walzer, 1970; Lubs and Ruddle, 1970; Bochkov et al., 1974; Jacobs et al., 1974; Hamerton et al., 1975; Nielsen and Sillesen, 1975). They have provided incidence figures for chromosome aberrations among the newborn populations necessary for genetic counseling. This report presents preliminary data on the frequency and type of major chromosome abnormalities in a newborn population observed in one hospital. Further detailed accounts particularly of clinical 
findings of abnormal infants will be reported elsewhere. This is the first contribution to the cytogenetic survey of the newborn population in Japan.

\section{MATERIALS AND METHODS}

A total of 2,795 infants were born alive in the Kitasato University Hospital during a period from 1 December 1975 to 30 November 1977. Out of 2,672 infants available for blood cultures, a successful cytogenetic analysis was possible in 2,626 infants consisting of 1,393 males and 1,233 females. Since 14,020 infants were delivered in Sagamihara during the period of the present study, and since $1,221(46.5 \%)$ of the 2,626 infants karyotyped in the Kitasato University Hospital were born to mothers resident in Sagamihara, this survey thus covered $8.7 \%$ of livebirths in Sagamihara.

Umbilical cord blood samples were collected in the delivery room immediately after birth and cell cultures were set up within 24 hours (48 hours at weekend). $0.3 \mathrm{ml}$ of whole blood were inoculated into a plastic culture tube containing $3 \mathrm{ml}$ of Eagle's Minimum Essential Medium supplemented with $15 \%$ of fetal calf serum and $0.1 \mathrm{ml}$ of phytohaemagglutinin. The containers were placed in a humidified gasflow incubator at $37^{\circ} \mathrm{C}$ for 3 days. Colchicinization, hypotonic treatment, fixation and preparation of slides were carried out according to standard procedures. Two slides were prepared from each baby, one of them was stained with conventional Giemsa and the other was stored for further special staining when requested. Repeat cultures were made with peripheral blood samples from cases with a chromosome abnormality, and at the same time blood samples were collected from the parents when needed. At least 5 cells were chromosomally analysed for each baby and one well-spread metaphase plate was photographed in each. When any doubtful finding was obtained about the chromosome, the cell-number for examination was increased. Special staining methods including Q-banding (Caspersson et al., 1970) and/or G-banding (Seabright, 1972) were applied to identify the abnormal chromosomes.

\section{RESULTS}

Some anamnestic and clinical data of the present population are given in Table 1. Of the 2,626 infants successfully karyotyped, 18 infants $(0.69 \%)$ were carriers of a major chromosome abnormality. In addition, 7 infants with a pericentric inversion of a chromosome 9 and one with a pericentric inversion of the $\mathrm{Y}$ chromosome were found in this series, but they were not included in the above 18 abnormal infants.

\section{Sex-chromosome abnormalities in phenotypic males}

Out of 1,393 male infants successfully karyotyped, 4 infants $(0.29 \%)$ showed sex-chromosome abnormalities: they were two (6/77 and 203/77) having a 47,XYY karyotype, one (394/76) showing a 46,XY/47,XYY (clone ratio 27:73) mosaicism and one $(389 / 76)$ carrying a $45, \mathrm{X} / 46, \mathrm{XY} / 47, \mathrm{XYY}$ (clone ratio 45:49:6) mosaicism. 
Table 1. Some anamnestic and clinical data of the study population.

(December 1, 1975 to November 30, 1977)

\begin{tabular}{lc}
\hline Total livebirths & 2,795 \\
No. of blood samples obtained & 2,672 \\
No. of unsuccessful cultures & 46 \\
No. of babies successfully karyotyped & 2,626 \\
$\quad$ Male & 1,393 \\
Female & 1,233 \\
Sex ratio & 1.13 \\
Mean maternal age (years) & $28.9 \pm 3.6$ \\
Mean gestational age (weeks) & $38.1 \pm 1.2$ \\
Mean birthweight (g) & \\
$\quad$ Male & $3,024.4 \pm 385.5$ \\
Female & $2,905.0 \pm 351.0$ \\
Mean birthlength (cm) & $48.8 \pm 1.9$ \\
Male & $48.1 \pm 1.8$ \\
Female & \\
Mean head circumference (cm) & $33.5 \pm 1.4$ \\
Male & $32.9 \pm 1.4$ \\
Female &
\end{tabular}

All the infants were physically normal at birth. Q-banding identification of the $Y$ chromosome was made in each of these infants.

\section{Sex-chromosome abnormalities in phenotypic females}

In 1,233 females karyotyped successfully, 4 infants $(0.32 \%)$ were those of sexchromosome abnormalities: they consisted of one case $(1224 / 76)$ of a $47, \mathrm{XXX}$ karyotype, one (327/76) showing a 45,X/47,XXX (clone ratio 50:50) mosaicism, one $(388 / 76)$ having a $45, \mathrm{X} / 46, \mathrm{XY} / 47, \mathrm{XYY}$ (clone ratio 38:57:5) mosaicism and one (1415/76) carrying a 45,X/46,XX (clone ratio 76:24) mosaicism. Case 388/76 and $389 / 76$ were twins with an apparent opposite sex. All of the above 4 infants were physically normal at birth. In case $388 / 76$, the $\mathrm{Y}$ chromosome was identified by the Q-banding method, and G-banding analyses were made for case 327/76, $1224 / 76$ and 1415/76. Cytogenetic data of infants with sex-chromosome abnormality are presented in Table 2 and their clinical data in Table 4.

\section{Autosomal trisomics}

Three $(316 / 77,699 / 77$ and 1243/77) were characterized by 21-trisomy with clinical features of Down's syndrome. The extra 21 chromosome was identified by the G-banding method in each.

\section{Unbalanced autosomal rearrangement and supernumerary chromosome}

One (346/76) had a 13-trisomy by translocation. The baby showed the clinical features characteristic of Patau's syndrome and expired soon after birth. The father of this baby was a carrier of a balanced 13/14 translocation. Another case (700/76) 
Table 2. Cytogenetic data of the 8 infants with sex chromosome abnormalities.

\begin{tabular}{|c|c|c|c|c|c|c|c|c|c|}
\hline \multirow{2}{*}{ Case No. } & \multirow{2}{*}{ Tissue } & \multicolumn{5}{|c|}{ Chromosome count } & \multirow{2}{*}{ Karyotype } & \multicolumn{2}{|c|}{ Karyotype } \\
\hline & & $<45$ & 45 & 46 & 47 & Total & & Mother & Father \\
\hline \multirow[t]{2}{*}{$327 / 76$} & Cord blood & - & 4 & - & 9 & 13 & \multirow{2}{*}{$45, \mathrm{X} / 47, \mathrm{XXX}$} & \multirow{2}{*}{$46, X X$} & \multirow{2}{*}{$46, X Y$} \\
\hline & Peripheral blood & 1 & 54 & 3 & 42 & 100 & & & \\
\hline \multirow[t]{2}{*}{$388 / 76$} & Cord blood & 2 & 17 & 27 & 1 & 47 & \multirow{4}{*}{$\begin{array}{c}45, X / 46, X Y / 47, \\
\text { XYY } \\
45, X / 46, X Y / 47, \\
\quad X Y Y\end{array}$} & \multirow{3}{*}{ - } & \multirow{2}{*}{ - } \\
\hline & Peripheral blood & - & 23 & 33 & 4 & 60 & & & \\
\hline \multirow[t]{2}{*}{$389 / 76$} & Cord blood & 2 & 42 & 51 & 5 & 100 & & & \\
\hline & Peripheral blood & - & 38 & 36 & 6 & 80 & & - & - \\
\hline \multirow[t]{2}{*}{$394 / 76$} & Cord blood & - & 1 & 4 & 11. & 16 & \multirow{2}{*}{$46, X Y / 47, X Y Y$} & \multirow{2}{*}{-} & \multirow{2}{*}{ - } \\
\hline & Peripheral blood & - & - & 27 & 73 & 100 & & & \\
\hline $1224 / 76$ & Cord blood & - & - & 2 & 18 & 20 & $47, \mathrm{XXX}$ & $46, X X$ & - \\
\hline \multirow[t]{2}{*}{$1415 / 76$} & Cord blood & 1 & 39 & 10 & - & 50 & \multirow{2}{*}{$45, X / 46, X X$} & \multirow{2}{*}{-} & \multirow{2}{*}{ - } \\
\hline & Peripheral blood & - & 36 & 14 & - & 50 & & & \\
\hline \multirow[t]{2}{*}{$6 / 77$} & Cord blood & - & - & 2 & 18 & 20 & \multirow{2}{*}{$47, X Y Y$} & \multirow{2}{*}{-} & \multirow{2}{*}{$46, X Y$} \\
\hline & Peripheral blood & - & - & 3 & 17 & 20 & & & \\
\hline \multirow[t]{2}{*}{$203 / 77$} & Cord blood & - & - & - & 10 & 10 & \multirow{2}{*}{$47, X Y Y$} & \multirow{2}{*}{ - } & \multirow{2}{*}{ - } \\
\hline & Peripheral blood & - & - & 3 & 17 & 20 & & & \\
\hline
\end{tabular}

had 47 chromosomes with an additional chromosome smaller than a member of $\mathrm{G}$ chromosomes $(47, \mathrm{XY},+$ mar). G- and Q-banding methods were not useful for the identification of this marker chromosome. The mother of this baby had 47 chromosomes with an additional chromosome which was similar in shape and size to that found in the baby. This baby and the mother were physically normal.

\section{Balanced autosomal rearrangements}

Among 2,626 infants successfully karyotyped, there were 5 infants $(0.19 \%)$ who had a balanced Robertsonian translocation. Three (1034/76, 1039/76 and 281/77) showed 13/14 translocation and two (737/76 and 1242/77) 14/22. Two (737/76 and 1034/76) were inherited from the father and three (1039/76, 281/77 and 1242/77) from the mother. The abnormal chromosomes were identified by the G-banding method in them. In case 737/76, a chromosome examination had been undertaken in amniotic fluid cells at the 18th week of gestation, since the mother had previously an abortion with trisomy 22 and the father had been known to be a balanced 14/22 translocation carrier. Cytogenetic data for the above cases are presented in Table 3 and their clinical data in Table 4.

\section{DISCUSSION}

The current literature refers to at least seven cytogenetic studies of unselected newborn infants populations (Sergovich et al., 1969; Gerald and Walzer, 1970; Lubs and Ruddle, 1970; Bochkov et al., 1974; Jacobs et al., 1974; Hamerton et al., 1975; Nielsen and Sillesen, 1975). Their data are summerized in Table 5 along with the 


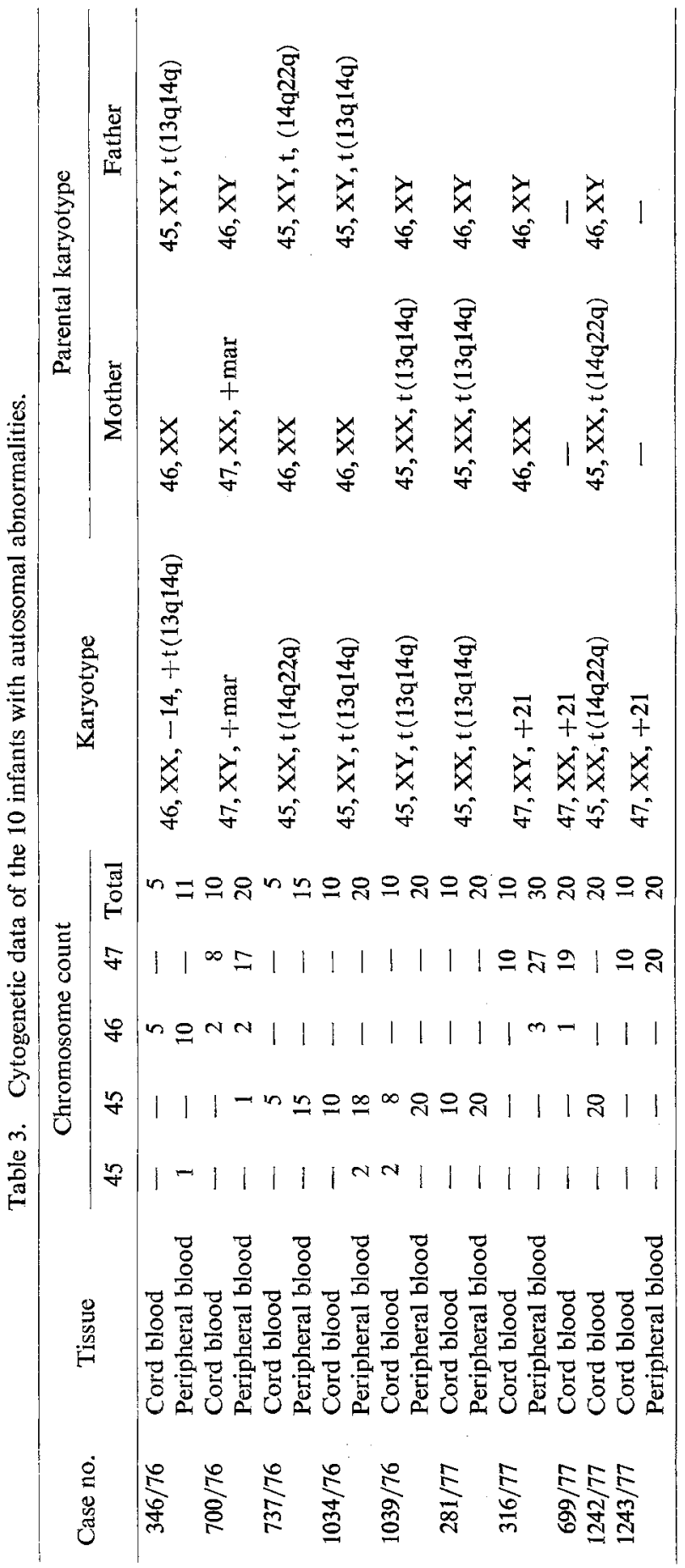




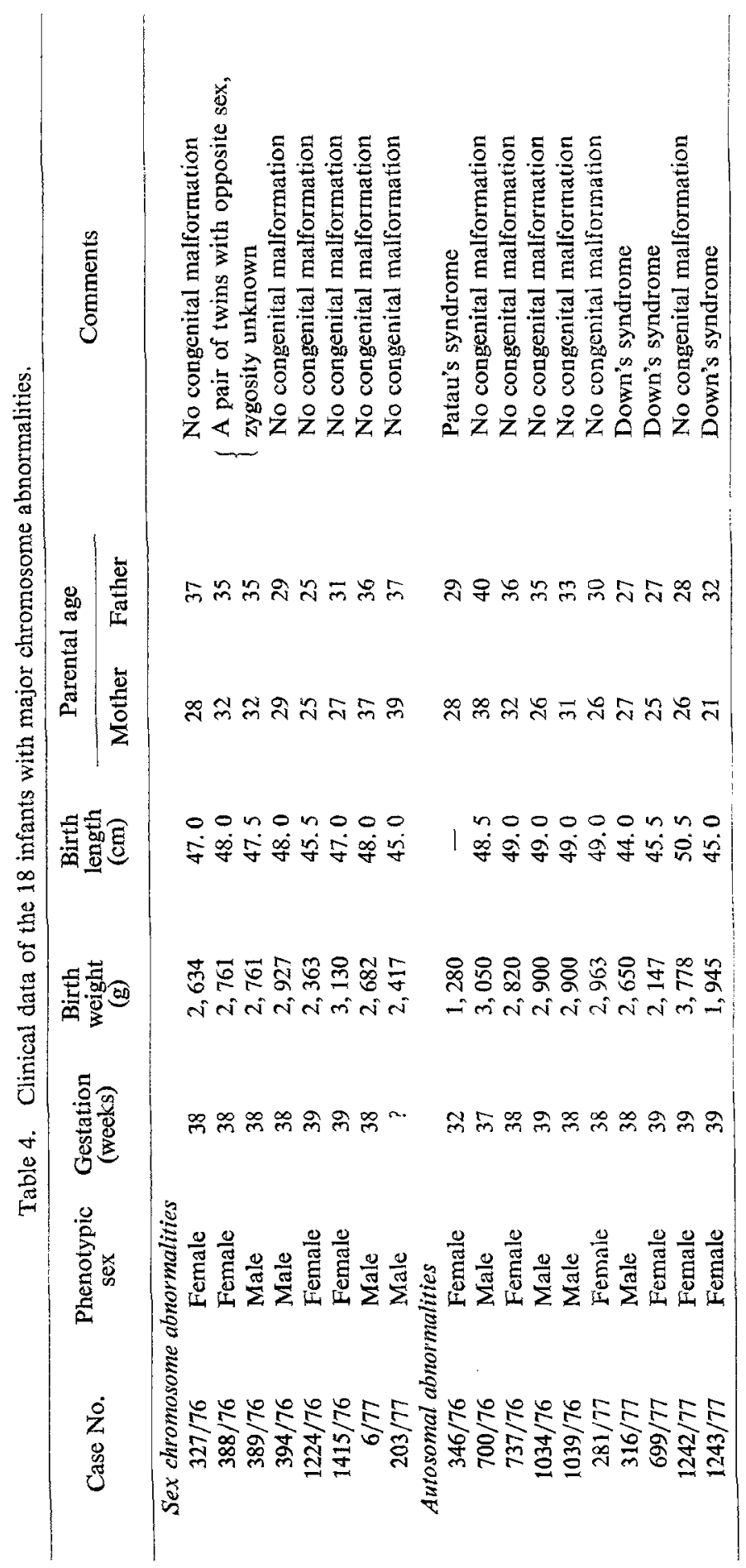


Table 5. Incidence of major chromosome abnormalities among newborn infants.

\begin{tabular}{|c|c|c|c|c|}
\hline \multirow{2}{*}{ Type of chromosome abnormalities } & \multicolumn{2}{|c|}{ Present study } & \multicolumn{2}{|c|}{ Previous 7 studies* } \\
\hline & Total & Per cent & Total & Per cent \\
\hline \multicolumn{5}{|l|}{ Sex-chromosome abnormalities } \\
\hline Nonmosaics & 3 & 0.11 & 88 & 0.18 \\
\hline Mosaics & 5 & 0.19 & 22 & 0.04 \\
\hline \multicolumn{5}{|l|}{ Autosomal abnormalities } \\
\hline Trisomics & 3 & 0.11 & 78 & 0.16 \\
\hline Balanced structural & 5 & 0.19 & 99 & 0.20 \\
\hline Unbalanced structural & 2 & 0.08 & 18 & 0.04 \\
\hline Total & 18 & 0.69 & 305 & 0.62 \\
\hline Total babies tested & 2,626 & & 49,244 & \\
\hline
\end{tabular}

* Sergovich et al. (1969); Gerald and Walzer (1970); Lubs and Ruddle (1970); Bochkov et al. (1974); Jacobs et al. (1974); Hamerton et al. (1975); Nielsen and Sillesen (1975).

present ones. The seven infant populations involve approximately 50,000 infants and the incidence of major chromosome abnormalities was shown as $0.62 \%$. There is a wide variation in the percentage of chromosomally abnormal neonates among them, the lowest at $0.46 \%$ (Hamerton et al., 1975) and the highest at $0.83 \%$ (Nielsen and Sillesen, 1975). The incidence of newborns with major chromosome abnormalities was $0.69 \%$ in the present study, though the sample size is still small for any assessment. Sex-chromosome abnormality occcurred in 8 infants $(0.30 \%), 4$ in phenotypic males and 4 in phenotypic females. Among the 8 sex-chromosome abnormalities, 5 were mosaics. While the incidence of sex-chromosome abnormalities found in our series was rather close to the averaged incidence of the seven reports, a marked difference occurs between them regarding mosaicism. The difference may be caused by the number of cells examined. Generally the diagnosis of mosaicism requires the analysis of a large number of cells. According to Bochkov et al. (1974), the probability of finding mosaics in $25 \%$ cells with the abnormal chromosome count was $76.1 \%$ in the initial 5-cell analysis. Nielsen (1975) reported that the first cell with the chromosome abnormality was found among the first 5 cells in 40 of the 44 cases with mosaicism. The incidence of female infants with abnormal sexchromosome constitutions in our series was almost identical to that of male infants. All infants with sex chromosome abnormalities in our series showed no physical abnormality. Of 10 infants with autosomal abnormalities, 6 were of the Robertsonian translocation type, one being unbalanced $13 / 14$, three with balanced $13 / 14$ and two showing balanced 14/22 type. The incidence of the balanced translocation was $0.19 \%$ in our series, which was comparable well to those in the seven other surveys. In a survey of 64 cases with $\mathrm{D} / \mathrm{D}$ translocation, Cohen (1971) found that $77 \%$ were of $13 / 14$ type. The excess of $13 / 14$ type among $\mathrm{D} / \mathrm{D}$ translocation was also observed in newborn surveys (Jacobs et al., 1974; Hamerton et al., 1975; Nielsen and Sillesen, 1975) and in our series. In contrast, 14/22 translocation was very rare, 
only one (Jacobs et al., 1974) having been reported in the seven newborn surveys. An unbalanced 13/14 translocation or 13-trisomy by translocation was also rare, only two (Sergovich et al., 1969; Jacobs et al., 1974) having been detected in the seven newborn surveys. It is of clinical importance to study the phenotypic effects of sex-chromosome abnormalities through follow-up studies of the infants with those abnormalities. Amniocentesis may be efficiently applied for familial members having a balanced Robertsonian translocation in preventing the birth of unbalanced zygotes in future pregnancies.

Acknowledgments We would like to thank Professor Emeritus Dr. Sajiro Makino, Hokkaido University, Sapporo, who read the manuscript and offered many valuable suggestions. We are indebted to the physicians and delivery-room staff of the Kitasato University Hospital, without whose continual efforts this investigation could not have been carried out.

\section{REFERENCES}

Bochkov, N.P., Kuleshov, N.P., Chebotarev, A.N., Alekhin, V.I., and Midian, S.A. 1974. Population cytogenetic investigation of newborns in Moscow. Humangenetik 22: 139-152.

Caspersson, T., Zech, L., Johansson, C., and Modest, E.J. 1970. Identification of human chromosomes by DNA-binding fluorescent agents. Chromosoma (Berl.) 30: 215-227.

Cohen, M.M. 1971. The chromosomal constitution of 165 human translocations involving D group chromosomes identified by autoradiography. Ann. Génét. 14: 87-96.

Gerald, P.S. and Walzer, S. 1970. Chromosome studies of normal newborn infants, in Human Population Cytogenetics (Jacobs, P.A., Price, W.H., and Law, P., eds.), Edinburgh University Press, Edinburgh, pp. 143-151.

Hamerton, J.L., Canning, N., Ray, M., and Smith, S. 1975. A cytogenetic survey of 14,069 newborn infants. Clin. Genet. 8: 223-243.

Jacobs, P.A., Melville, M., Ratcliff, S., Keay, A.J., and Syme, J. 1974. A cytogenetic survey of 11,680 newborn infants. Ann. Hum. Genet. 37: 359-376.

Lubs, H.A. and Ruddle, F.H. 1970. Chromosome abnormalities in the human population: Estimation of rates based on New Haven newborn study. Science 169: 495-497.

Nielsen, J. 1975. Chromosome mosaicism in a population sample. Humangenetik 29: 155-159.

Nielsen, J. and Sillesen, I. 1975. Incidence of chromosome aberrations among 11148 newborn children. Humangenetik 30: 1-12.

Seabright, M. 1972. The use of proteolytic enzymes for the mapping of structural rearrangements in the chromosomes of man. Chromosoma (Berl.) 36: 204-210.

Sergovich, F., Valentine, G.H., Chen, A.T.L., Kinch, R.A.H., and Smout, M.S. 1969. Chromosome aberrations in 2159 consecutive newborn babies. N. Engl. J. Med. 280: 851-855. 